\title{
Relativistic Fermi-Ulam Map: Application to WEGA Stellarator Lower Hybrid Power Operation*
}

\author{
V. Fuchs ${ }^{1}$, H. P. Laqua ${ }^{2}$ J. Seidl ${ }^{1}$, L. Krlín ${ }^{1}$, R. Pánek ${ }^{1}$, J. Preinhaelter ${ }^{1}$, J. Urban ${ }^{1}$ \\ ${ }^{1}$ Institute of Plasma Physics AS CR, v.v.i., Prague, CZ, Association EURATOM/IPP.CR \\ ${ }^{2}$ Max Planck Institute für Plasmaphysik, EURATOM Association, D-17491 Greifswald, Germany
}

Analytical and numerical support is here provided in support of the explanation ( $\mathrm{H}$. Laqua et al., submitted to Plasma Physics and Controlled Fusion) for the observation of $\sim \mathrm{MeV}$ electrons during Lower Hybrid (LH) operation in EC pre-heated plasma at the WEGA stellarator [M. Otte, H. P. Laqua, E. Chlechowitz, S. Marsen, J. Preinhaelter, T. Stange, A. Rodatos, J. Urban and D. Zhang, Nukleonika, 57 (2012) 171]. In the quoted experiments, LH power from the WEGA TE 11 circular waveguide, $9 \mathrm{~cm}$ diameter, un-phased, $2.45 \mathrm{GHz}$ antenna, is radiated into a $\mathrm{B} \cong 0.5 \mathrm{~T}, \overline{\mathrm{n}}_{\mathrm{e}} \cong 5 \times 10^{17} 1 / \mathrm{m}^{3}$ plasma at $\mathrm{T} \cong 10 \mathrm{eV}$ bulk temperature with an EC-generated $50 \mathrm{keV}$ population of electrons. In response, the fast electrons travel around flux or drift surfaces essentially without collisions, repeatedly interacting with the rf field close to the antenna mouth, and gaining energy in the process. Our WEGA antenna calculations indicate a predominantly standing electric field pattern at the antenna mouth. From a simple approximation of the corresponding Hamiltonian equations of motion we derive here a relativistic generalization of the simplified area-preserving Fermi-Ulam (F-U) map [Lieberman and Lichtenberg, Phys. Rev. A5 (1972) 1852), Lichtenberg, Lieberman, and Cohen, Physica 1D (1980) 291], allowing phase-space global stochasticity analysis. At typical WEGA plasma and antenna conditions, and with correlated phases between electron - antenna electric field interaction events, the F-U map and supporting numerical simulations predict an absolute energy barrier in the range of $300 \mathrm{keV}$. In contrast, with random phases intervening between interaction events the electron energy can reach $\sim \mathrm{MeV}$ values, compatible with the measurements on WEGA.

Keywords: stellarator, lower hybrid, current drive, Hamiltonian, electron, stochastic.

PACS: 52.55.Hc, 52.50.-b, 52.20.Dq, 52.50.Gj, 52.50.Sw, 05.40.-a

\section{INTRODUCTION}

High energy electrons in the $\mathrm{MeV}$ range were observed by Laqua et al. ${ }^{1}$ during lower hybrid (LH) operation in EC pre-heated plasma at the WEGA stellarator ${ }^{2,3}$. The explanation given by Laqua et $\mathrm{al}^{\mathbf{1}}$ for the high electron energies is their stochastic acceleration resulting from repeated interaction with the LH standing wave electric field at the antenna mouth, as the electrons circulate on drift surfaces around the torus. In order to provide a theory framework for understanding this interaction, we derive and analyze here a relativistic generalization of the socalled simplified Fermi-Ulam process, given in its original form by Lieberman and Lichtenberg ${ }^{4}$, and Lichtenberg, Lieberman, and Cohen 5 .

Stochastic (or, synonymously, chaotic) acceleration of charged particles is a subject of considerable interest for the understanding of many phenomena in astrophysical as well as 
laboratory plasmas. Particle motion can be rendered stochastic in interaction with waves, shocks, turbulence, i.e. with plasma and/or electric and/or magnetic field perturbations. The subject was initiated in a few pioneering papers by Fermi ${ }^{6,}{ }^{7}$, Davis ${ }^{8}$, and Kulsrud and Ferrari ${ }^{9}$, to give a representative sample. In his first paper: Fermi $^{6}$ discusses interactions of particles with surrounding media fluctuations, turbulence in general. Such stochastic acceleration processes are referred to, at least in the astrophysical literature (e.g. Petrosian ${ }^{\mathbf{1 0}}$ ), as Fermi processes of the $2^{\text {nd }}$ order. In his second paper Fermi ${ }^{7}$ introduces particle chaos and acceleration caused by singular impact events, shocks or collisions with spatially separated entities, such as magnetically charged walls etc., usually referred to as Fermi processes of the $1^{\text {st }}$ order. Davis ${ }^{\mathbf{8}}$ and Kulsrud and Ferrari ${ }^{\mathbf{9}}$ underlined the diffusive nature of $2^{\text {nd }}$ order Fermi processes and developed their kinetic description. Much work has been devoted in the past to relativistic chaos caused by magnetic field perturbations of respectively proton or electron beams in particle accelerators (e.g., Chao et al. ${ }^{11}$ ) and free electron lasers (e.g., Chen and Davidson ${ }^{12}$ ). In either case, the particles are relativistic to start with, the magnetic field perturbation enters the Hamiltonian via the vector potential, and the beyond a certain interaction parameter threshold the magnetic perturbation acts to scatter particles away from the device axis, where the particle orbits are integrable, thereby deteriorating the device performance. An important non-relativistic variation on the particle chaos caused by magnetic perturbations in accelerators and FELs, are resonant magnetic perturbations $^{13}$ deliberately excited at the tokamak edge in order to mitigate the effects of edge localized modes (ELMs). Wave-particle interactions are a special case of a $2^{\text {nd }}$ order Fermi process; a novel treatment of this particular problem has been recently undertaken by Kominis, Ram and Hizanidis ${ }^{14}$.

The present work deals with a special case of $1^{\text {st }}$ order Fermi acceleration, specifically with electron interacting (or "colliding") with a spatially localized electromagnetic wave-packet. The motivation for the work is driven by the wish to provide theory support to the analysis of the observation of high energy $\mathrm{MeV}$ electrons during Lower Hybrid (LH) operation in EC preheated plasma at the WEGA stellarator, reported by the WEGA team ${ }^{1}$. To do so, we first extend previous work by Fuchs et al. ${ }^{15}$, Pavlo and Krlin. ${ }^{16}$, Pánek ${ }^{17}$, Krlin et al. ${ }^{18}$, and Seidl ${ }^{19}$ on electron phase space stochasticity and acceleration caused by the electrons repeatedly passing through a spatially localized travelling lower hybrid (LH) wave, to the case of electrons passing through a standing wave. An important distinction between the travelling and standing wave cases is that 
for a single passage through the wave region, the electron Hamiltonian is conserved in the travelling wave case, and remains so even for relativistic velocities, as was shown by Seidl ${ }^{19}$. In either case, electron orbits tend to become stochastic as a result of the phase lag accumulated on the field free path between successive interaction events. Second, we show that the standing wave interaction in question can be approximated by the "simplified" version of the Fermi-Ulam map (Lieberman and Lichtenberg ${ }^{4}$, Lichtenberg et al..$^{5}$ ), which we generalize to relativistic velocities.

In addition to the orbiting electron - travelling wave interaction, mentioned above, the present WEGA problem is also closely related to the problem of tokamak edge electrons interacting of with a LH wave spectrum ${ }^{20-25}$. A Fermi-like non-relativistic map was developed (Fuchs et al. ${ }^{21}$, Goniche et al. ${ }^{22}$ ) to describe the latter interaction, which occurs as the electrons travel along magnetic field lines in front of the lower hybrid grill. In the process, the electrons tend to diffuse to higher energies and depending on the radiated wave electric field strength will reach an upper limit given by the extent of Chirikov ${ }^{\mathbf{2 6}}$ resonance (i.e. spectrum Fourier mode) overlap. The LH grill waveguide phasing (typically $\pi / 2$ ) is the underlying cause for the electron stochastic acceleration from their cold edge value of typically $10-20 \mathrm{eV}$ up to few $\mathrm{keV}$. In the orbiting electron problem, of interest here, the phase lag acquired by the electron on the fieldfree part of its orbit is the counterpart of the grill waveguide phasing.

As already mentioned, we show here that the interaction of electrons with the WEGA lower hybrid electric field at the antenna mouth is Fermi-like. The Fermi-Ulam approximation ${ }^{4,5}$ of the $1^{\text {st }}$ order Fermi process to which we reduce the electron equations of motion, has received much attention in the past (Lichtenberg and Lieberman ${ }^{27}$, and further references therein), not only because it is a conveniently simple area-preserving map, but because it serves to represent nearintegrable Hamiltonian systems with two degrees of freedom. The simplified F-U model describes a particle bouncing between two fixed surfaces, one of which delivers momentum of some prescribed temporal oscillating nature to the particle. To the best of our knowledge, most of the past work related to the F-U map is limited to non-relativistic conditions with the notable exception of the gravitational bouncer model (Pustylnikov ${ }^{\mathbf{2 8}}$ ) in which the particle returns by gravity to an oscillating surface. The particle energy in Pustylnikov's model can grow without limits, while in the present relativistic F-U model the particle energy is limited by period-1 fixedpoint stability, in similar way as in the usual non-relativistic case ${ }^{5,27}$, but with a condition for 
global stochasticity onset modified by relativity. The issue here is that in order for an electron to reach $\mathrm{MeV}$ values under WEGA conditions, we find that random phases between interaction events have to be assumed.

To set up the Hamiltonian for the WEGA orbiting electron interaction problem, we determine the electromagnetic power radiated at $\mathrm{f}_{\mathrm{LH}}=2.45 \mathrm{GHz}$ into a $\mathrm{T}_{\mathrm{e}} \cong 10 \mathrm{eV}$, line average $\overline{\mathrm{n}}_{\mathrm{e}} \cong 5 \times 10^{17}$ $1 / \mathrm{m}^{3}, \mathrm{~B} \cong 0.5 \mathrm{~T}$, edge plasma by the WEGA $\mathrm{TE}_{11}$ circular waveguide, $9 \mathrm{~cm}$ diameter, un-phased antenna with a double cut at its front (Podoba et al. ${ }^{29}$ ). The antenna was modeled as a two waveguide grill with zero phasing and calculations were carried out with the full wave coupling code described in Preinhaelter et al. ${ }^{30}$. The results indicate that at the given conditions the electric field is polarized predominantly in the $\mathrm{z}$ (toroidal) direction; with $40 \%$ of the power reflected. Most of the transmitted power ends up in non-propagating eigenmodes, only $10 \%$ propagates in opposite directions along resonance cones, which due to the lack of phasing also forms a standing wave pattern at the antenna mouth. Fig. 1 illustrates the resonance cone structure of the propagating portion of the $E_{z}$ field, and Fig. 2 shows the field $E_{z}$ and its spectrum close to the antenna mouth. The $E_{z}$ field is predominantly electrostatic, so the vector potential from the Hamiltonian (1) below can be omitted.

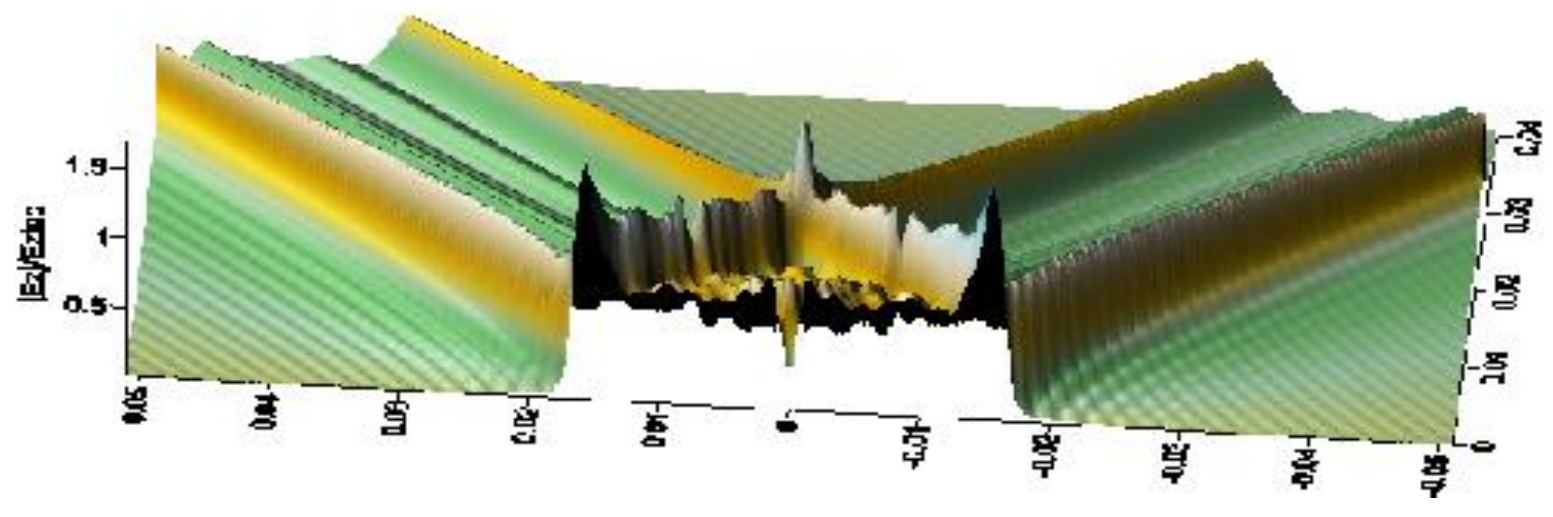

FIG 1 WEGA antenna $E_{z}$ field: resonance cone structure. 

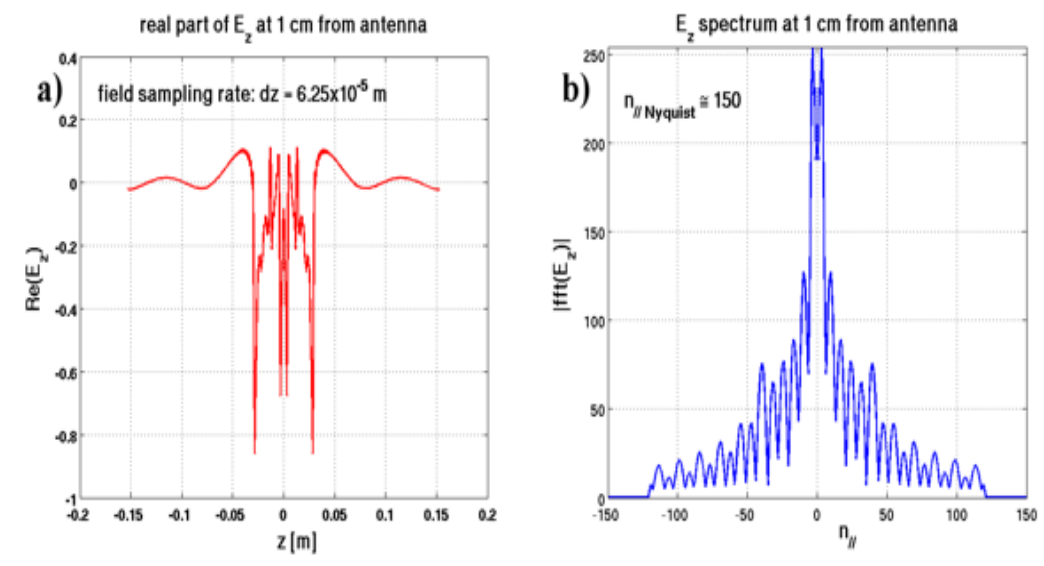

FIG. 2 WEGA antenna $E_{z}$ field: a) Re(Ez) along the antenna in the toroidal direction, at $1 \mathrm{~cm}$ from the antenna mouth, b) $E_{z}$ spectrum as function of $n / /$.

The prominent low-n// peak seen in FIG. 2b is the reason for the dominant standing wave character of the $\mathrm{E}_{\mathrm{z}}$ field near the antenna.

Fast electrons $(\geq 50 \mathrm{keV})$ cycle essentially without collisions around flux or drift surfaces, shown in Fig. 3:

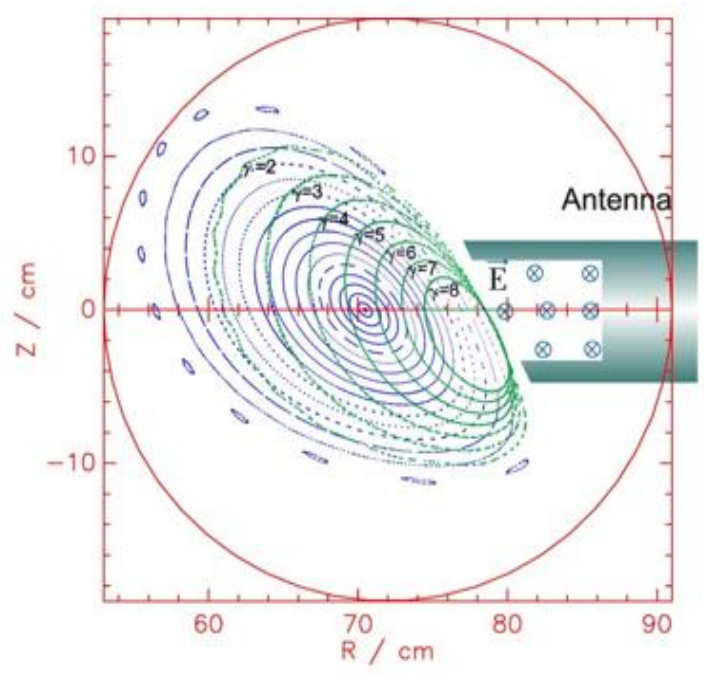

FIG. 3 Flux surfaces (blue) and particle drift surfaces (green) for different relativistic $\gamma$-factors. We see a simplified sketch of the antenna field. This is a poloidal cut of a toroidal configuration. The antenna is inserted beyond the last closed flux surface so the electrons circulate freely. (Reproduced from Laqua et al. $\left.{ }^{1}\right)$ 
The dominant collision frequency $v_{\text {coll }}$ is that of the fast $\left(\mathrm{T}_{\text {hot }} \geq 50 \mathrm{keV}\right)$ electrons on the thermal bulk electrons $\left(\mathrm{T}_{\mathrm{e}} \cong 10 \mathrm{eV}, \mathrm{n}_{\mathrm{e}} \cong 5 \times 10^{17} 1 / \mathrm{m}^{3}\right)$, which gives $v^{\text {hot/thermal }} \leq 4 \mathrm{~s}^{-1}$, so the collision time $\tau_{\text {coll }}$ as the electron speeds up is always larger than 0.25 seconds. This is to be compared with the electron travel time $\tau_{\text {trans }}=\mathrm{L} / \mathrm{v}(\mathrm{L}=2 \pi \mathrm{qR})$ around a flux or drift surface, which is always smaller than $\mathrm{L} / \mathrm{c} \cong 7.5 \times 10^{-8} \mathrm{sec}$. We take $\mathrm{R}=0.72 \mathrm{~m}$, and on the basis of results from the WEGA equilibrium code ${ }^{31}$ shown in Fig. 4 we estimate the safety factor to be $q \cong 5$. All this goes to show that collisions are most likely not the process which leads to randomizing phases on the field-free electron orbit.
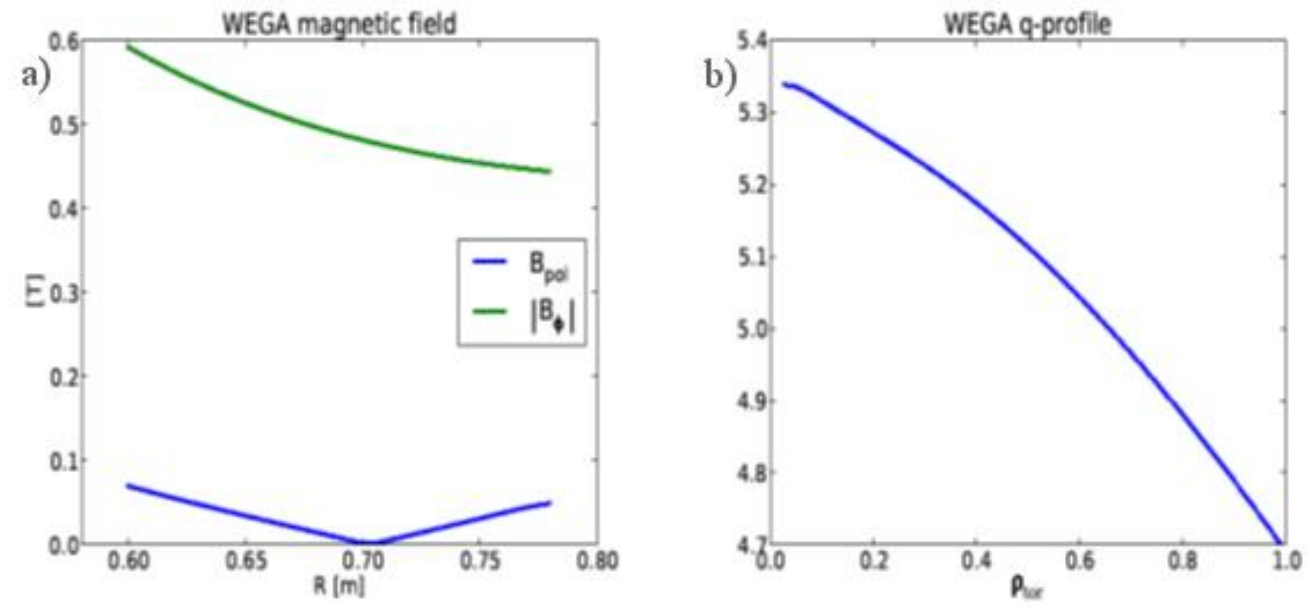

FIG. 4 a) Magnetic equilibrium of the experiment described in Laqua et al. ${ }^{1}$, determined from the equilibrium code in Otte et al. ${ }^{23}$. b) Safety factor corresponding to $\mathrm{B} \cong 0.5 \mathrm{~T}$ of the respective experiment is $\mathrm{q} \cong 5$. $\rho_{\text {tor }}$ is the normalized square root of toroidal flux.

The negligible collisionality in the low density WEGA plasma with an EC generated $50 \mathrm{keV}$ component allows an electron to repeatedly interact with the rf field close to the antenna mouth. The evolution of electron energy depends on whether its phases between interaction events are correlated or random. Essentially, for correlated phases the electron energy stochastic region is bounded, for random phases we find that the energy grows with increasing number of interaction events (i.e. with the number of cycles around drift surfaces). We support these findings by numerical as well as theory analysis. In order to better understand the nature of the interaction 
along with the resulting global stochasticity bound in the case of correlated phases, we show, in section II, that a simple approximation of the equations of motion leads to a relativistic version of the area-preserving Fermi-Ulam map, for which we also establish a relativistic version of the period-1 fixed point stability criterion. The present form of the relativistic F-U map is distinct from a previous relativistic F-U model - the gravitational bouncer model ${ }^{29}$, in which the particle energy can increase indefinitely even for correlated phases between interaction events. In section III we apply the theory of section II to WEGA experimental conditions of Laqua et al. ${ }^{1}$. From numerical and period-one fixed point stability analysis ${ }^{\mathbf{4}, 5}$, we find that the electron energy, for an electric field strength $50 \mathrm{kV} / \mathrm{m}$ (corresponding to about $10 \mathrm{~kW}$ of power radiated by the antenna), does not exceed at WEGA conditions about $300 \mathrm{keV}$. In order to reach electron energies in the $\mathrm{MeV}$ range, random phases between the electron-antenna interaction events therefore need to be assumed. Further in the WEGA context, it is interesting to note that Laqua et al. ${ }^{1}$ and Otte et al. ${ }^{2}$ report that at energies above $200 \mathrm{keV}$, the electron confinement in WEGA becomes highly asymmetric with electrons in one direction along drift surfaces suffering loss, so during LH operation a non-inductive current is a fortiori generated. First moments of the electron distribution obtained from the iterated F-U map give an LH current density of about $7 \mathrm{kA} / \mathrm{m}^{2}$, an LH driven current $\cong 230 \mathrm{~A}$, and dissipated LH power $\cong 225 \mathrm{~W}$. This compares favorably with results of the experiment ${ }^{1,2}$. In section IV, the principal phase space features, e.g. energy stochastic boundaries and diffusion coefficient, found using the relativistic F-U approach, are verified by symplectic integrations of the relativistic electron equations of motion. Finally, in section $\mathrm{V}$ we give our summary and conclusions.

\section{THE RELATIVISTIC FERMI-ULAM MAP}

In view of expected $\mathrm{MeV}$ electron energies we describe electron interaction with the standing wave field by a relativistic non-conservative and non-autonomous Hamiltonian with canonical momentum $\mathrm{P}=\mathrm{p}-\mathrm{e} \mathrm{A}_{\mathrm{z}}$, vector potential $\mathrm{A}_{\mathrm{z}} \simeq 0$, scalar potential $\Phi(\mathrm{z}, \mathrm{t})=\Phi(\mathrm{z}) \sin (\omega \mathrm{t})$, coordinate $\mathrm{z}$, rest mass $m_{e}$, and $\omega=2 \pi f_{L H}$ :

$$
H=m_{e} c^{2}(\gamma-1)+\Phi(z) \sin (\omega t), \quad \Phi(z)=-\left(e E_{0} / k\right) \sin (k z)
$$

The corresponding equations of motion 


$$
\frac{d p}{d t}=-\frac{\partial H}{\partial z}=-\sin (\omega t) \frac{\partial \Phi}{\partial z} ; \quad \frac{d z}{d t}=\frac{\partial H}{\partial p}=\frac{p}{m_{e} \gamma} ; \quad \gamma=\sqrt{1+\left(\frac{p}{c m_{e}}\right)^{2}}
$$

describe interaction with the field $\Phi(\mathrm{z}, \mathrm{t})$ at the antenna, where $\partial \Phi / \partial \mathrm{z}=\mathrm{E}_{\mathrm{z}}$ of FIG. 2. On the fieldfree orbits around a flux (or drift) surface, the auxiliary condition $\Phi \equiv 0$ applies.

We now show that under particular conditions of the WEGA experiment, the dynamical problem specified by Eqs (1) and (2) can be reduced to a relativistic form of the Fermi-Ulam process. For correlated phases between interaction events this allows analysis of the stochastic electron phase space. The fast electrons $(>50 \mathrm{keV})$ do not precisely sample the electric field variations since with increasing velocity their transit time $\left(10^{-9} \mathrm{~s}\right.$ at $\left.50 \mathrm{keV}\right)$ through the field region becomes shorter than the field period $1 / f_{\mathrm{LH}}=4 \times 10^{-10} \mathrm{~s}$. Denote therefore by $\mathrm{z}_{0}=0$ the position of the antenna, by $\mathrm{t}_{0}$ the time during an orbit at which interaction occurs and introduce the phase $\psi=\omega t$. Integrating Eqs (2) through the integration region and one field-free orbit then gives

$$
\begin{aligned}
& p_{1}-p_{0}=\left(e E_{0} / \omega\right) \int_{t} \sin (\psi) \cos [k z(\psi)] \delta\left(\psi-\psi_{0}\right) d \psi=m_{e} v_{q} \sin \left(\psi_{0}\right) \cos \left(k z_{0}\right) \\
& z_{1}-z_{0} \equiv L=\frac{p_{1}}{m_{e} \gamma_{1}}\left(t_{1}-t_{0}\right) ; \quad v_{q}=\frac{e E_{0}}{\omega m_{e}}
\end{aligned}
$$

where $\mathrm{E}_{0} \cong 0.56 \mathrm{kV} / \mathrm{cm}$ is the lower hybrid $\mathrm{E}_{\mathrm{z}}$-field amplitude in the quoted WEGA experiment ${ }^{1,2}$. With $\mathrm{z}_{0}=0$, recalling that $\mathrm{L}$ is the electron orbit length and introducing normalized momentum $\mathrm{u}=\mathrm{p} /\left(\mathrm{m}_{\mathrm{e}} \mathrm{v}_{\mathrm{q}}\right)$, Eqs (3) reduce to a normalized relativistic form of the F-U map

$$
u_{n+1}=u_{n}+\sin \left(\psi_{n}\right) ; \quad \psi_{n+1}=\psi_{n}+\frac{2 \pi M \gamma_{n+1}}{u_{n+1}} ; \quad M=\frac{L f}{v_{q}}
$$

The F-U map is a resonant system with the principal, so-called period 1 resonances ${ }^{5,27}$, occurring when the electron orbit time L/v equals an integer multiple of the field period 1/f, i.e. when $\mathrm{Lm}_{\mathrm{e}} \gamma / \mathrm{p}=\mathrm{n} / \mathrm{f}, \mathrm{n}=1,2,3, \ldots$

We emphasize that the condition for applicability of the Fermi-Ulam model to the WEGA situation is limited to fast electrons whose transit time through the interaction region is shorter than the electric field period $1 / \mathrm{f}_{\mathrm{LH}}$. This issue will be addressed in more detail in section $\mathrm{V}$, where symplectic integrations of the equations of motion (2) are carried out. 


\section{Global Stochasticity Boundary from Period 1 Momentum}

A usual method of identifying and analyzing phase space stochasticity of various maps, including the Fermi-Ulam process, is to locally represent the map by the "Standard" map whose stochasticity properties are well known ${ }^{27},{ }^{28}$. We thus linearize (4) around the period 1 momentum $\mathrm{u}_{1}=\mathrm{M} \gamma / \mathrm{n}, \mathrm{n}=1,2,3 \ldots$ Let therefore $\mathrm{u}=\mathrm{u}_{1}+\Delta \mathrm{u}$, shift the phase $\psi=\Theta-\pi / 2$, and introduce a new action variable $\mathrm{I}=\mathrm{K} \Delta \mathrm{u}$, where $\mathrm{K}=2 \pi \mathrm{M} / \gamma_{1}\left(\mathrm{u}_{1}\right)^{2}$ is the stochasticity, or interaction, parameter [we note that the usual non-relativistic form of the interaction parameter is $K=2 \pi \mathrm{M} /\left(\mathrm{u}_{1}\right)^{2}$, where $\mathrm{u}_{1}=\mathrm{v}_{1} / \mathrm{v}_{\mathrm{q}}$ and $\mathrm{v}_{1}$ is the period 1 velocity]. The Lorentz factor in terms of $\mathrm{u}_{1}$ is $\gamma_{1}=\sqrt{ } 1+\alpha\left(\mathrm{u}_{1}\right)^{2}$, $\alpha=\left(v_{\mathrm{q}} / \mathrm{c}\right)^{2}$. The map (4) is thus locally represented by the Chirikov-Taylor Standard map ${ }^{27,28}$

$$
I_{n+1}=I_{n}+K \sin \left(\Theta_{n}\right) ; \quad \Theta_{n+1}=\Theta_{n}+I_{n+1}
$$

which exhibits global stochasticity when $K \geq 1$, i.e. when $2 \pi M / \gamma_{1}\left(u_{1}\right)^{2} \geq 1$. This leads to a global stochasticity upper bound $\mathrm{x} \equiv\left(\mathrm{u}_{1}\right)^{2}$ given by the cubic equation

$$
\alpha x^{3}+x^{2}-(2 \pi M)^{2}=0 ; \quad \alpha=\left(v_{q} / c\right)^{2}
$$

\section{APPLICATION OF THE FERMI-ULAM MAP TO WEGA}

We now apply results of the preceding section to the WEGA LH operation conditions ${ }^{\mathbf{1}}$ : electron orbit mean radius (from Fig. 3) $\mathrm{R} \cong 0.72 \mathrm{~m}, \mathrm{~B} \cong 0.5 \mathrm{~T}$. An important factor entering into the interaction parameter $M$ is the electron orbit length $L=2 \pi R q=22.6 \mathrm{~m}$. In order to indicate how the stochasticity upper bound scales with electric field strength $E_{0}$, we solve Eq. (6) in the range $\mathrm{E}_{0} \in<10^{4}, 5 \times 10^{5}>\mathrm{kV} / \mathrm{cm}$. At WEGA conditions and in the given range of electric field, Eq. (6) has one real root, leading to the energy bound shown in Fig 5a. Figure 5b is then produced at $\mathrm{E}_{0}=50 \mathrm{kV} / \mathrm{m}$, close to the field value quoted in the experiment ${ }^{1}$, and indicates a saturation of the energy response as the electron speeds up 

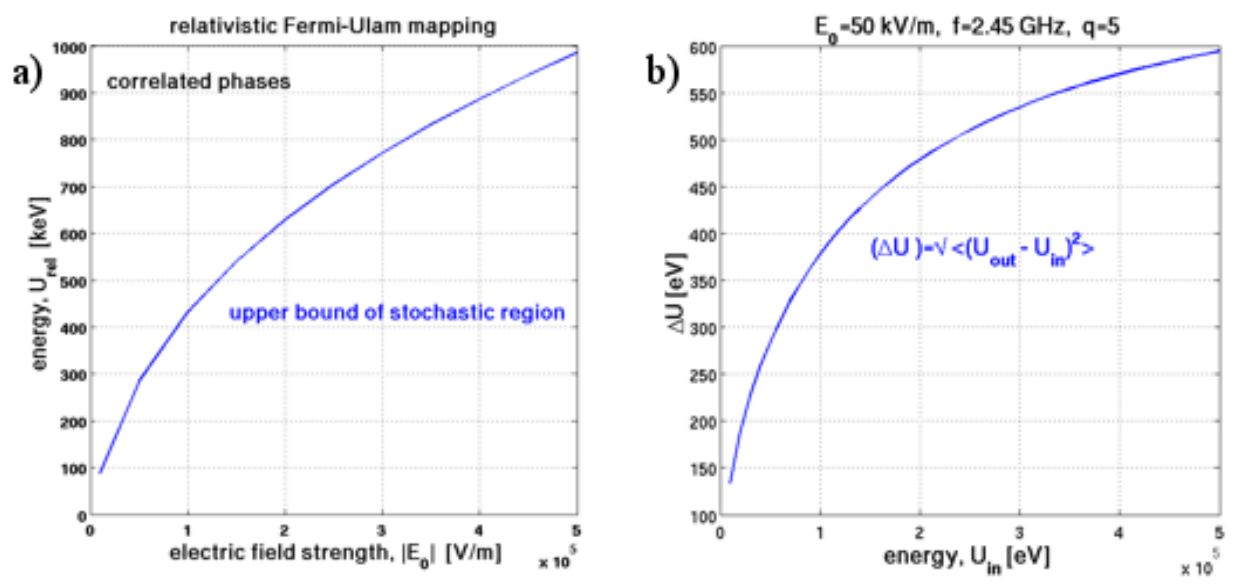

FIG. 5 a) Upper bound $U_{\text {rel }}$ of the relativistic F-U map (4), at WEGA conditions ${ }^{1}$, as function of electric field $\left.E_{0} . b\right)$ Electron energy jumps $U_{\text {out }}-U_{\text {in }}$ between iterations of the F-U map (4), as function of $U_{\text {in }}$.

Specifically, at $\mathrm{E}_{0} \cong 50 \mathrm{kV} / \mathrm{m}$, we have $\mathrm{v}_{\mathrm{q}} \cong 5.7 \times 10^{5} \mathrm{~m} / \mathrm{s}$, the interaction parameter is $\mathrm{M}=9.7 \times 10^{4}$, giving the real solution $\mathrm{x}=3.9 \times 10^{5}$ of Eq. (6). The corresponding stochasticity threshold is $\mathrm{U}_{1}=512\left(\gamma_{1}-1\right)=284 \mathrm{keV}$, where $\gamma_{1}=\sqrt{ } 1+\alpha \mathrm{x}$. To see how this predicted energy threshold compares with maximum energy obtained directly from the map (4), we carry out $10^{6}$ iterations of the map for 100 electrons having initially identical energy $(50 \mathrm{keV})$ but initially distributed with random phases. The resulting energy histogram is shown in Fig 6a. For a much higher value of electric field, say $250 \mathrm{kV} / \mathrm{m}$, the predicted stochastic bound from Eq. (6) is $705 \mathrm{keV}$, and the associated maximum energy histogram is shown in FIG. $6 \mathbf{b}$.
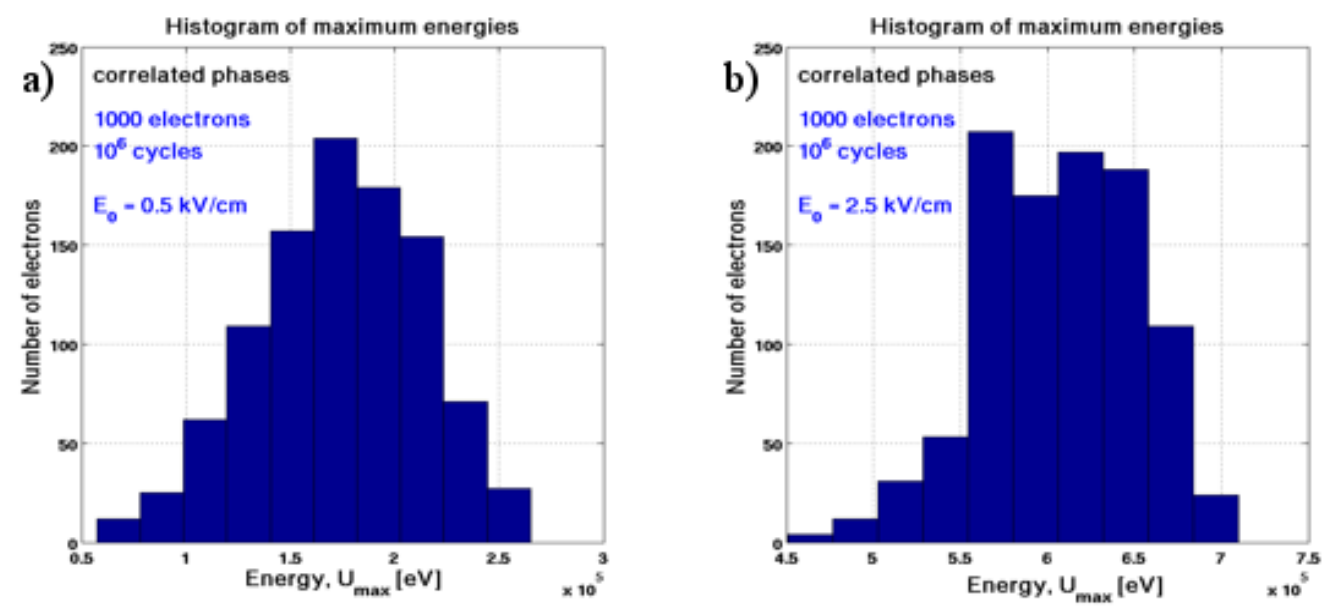
FIG. 6 Histogram of maximum energies in an ensemble of 100 electrons distributed with random initial phases. Phases between the $10^{6}$ interaction events are correlated, i.e. non-random. a): $E_{0}=50 \mathrm{kV} / \mathrm{m}, \mathrm{b}$ ) $\mathrm{E}_{0}=250 \mathrm{kV} / \mathrm{m}$.

In both cases we see that the most energetic electrons of the distributions indeed lie close to the predicted stability theory values of $284 \mathrm{keV}$, and $705 \mathrm{keV}$, respectively. A surface of section plot for an electron at the high end of the energy range for $E_{0}=50 \mathrm{kV} / \mathrm{m}$ shown in FIG. 7 clearly indicates the expected global stochasticity for $U<U_{\max }$. The maximum energy at $50 \mathrm{kV} / \mathrm{m}$ is rather short of the expected $\mathrm{MeV}$ range, which possibly suggests that the phases of particles between interaction events are random.
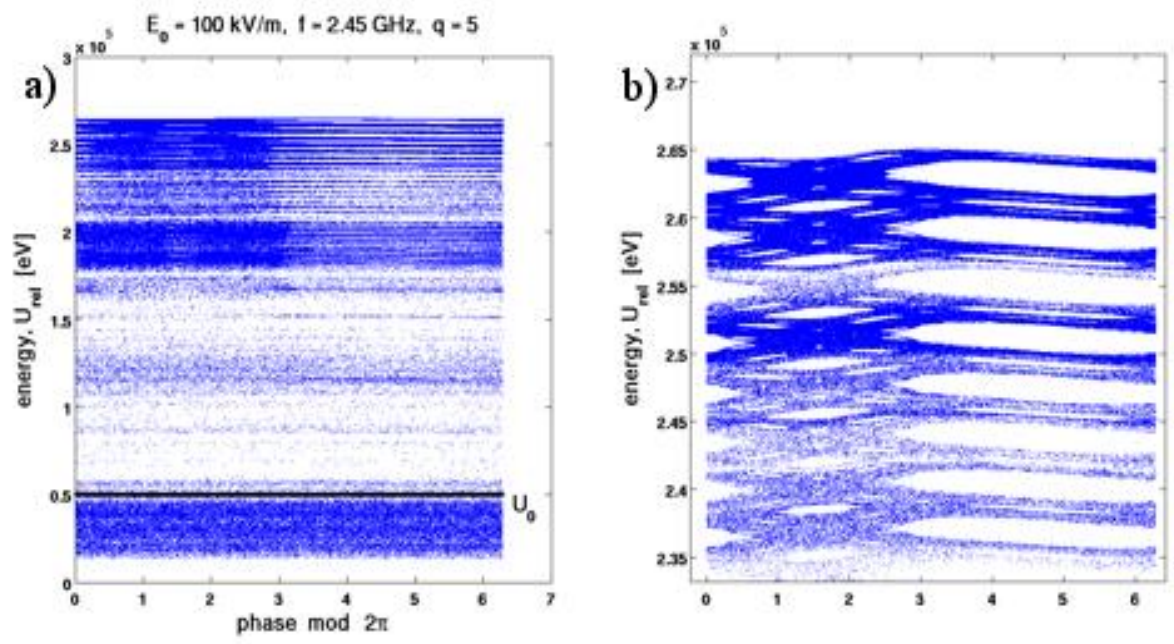

FIG. 7 a) Surface of section, energy versus phase, from the Fermi-Ulam map (4) at WEGA operating conditions from Laqua et al. ${ }^{1}$. Phases between interaction events are assumed correlated. $\mathrm{U}_{0}$ indicates the energy initial condition $(50 \mathrm{keV})$. b) Blow-up of the stochasticity boundary shows the lowest-order resonances.

Finally, FIG. 8 demonstrates the critical effect of random phases on the energy of circulating electrons: 


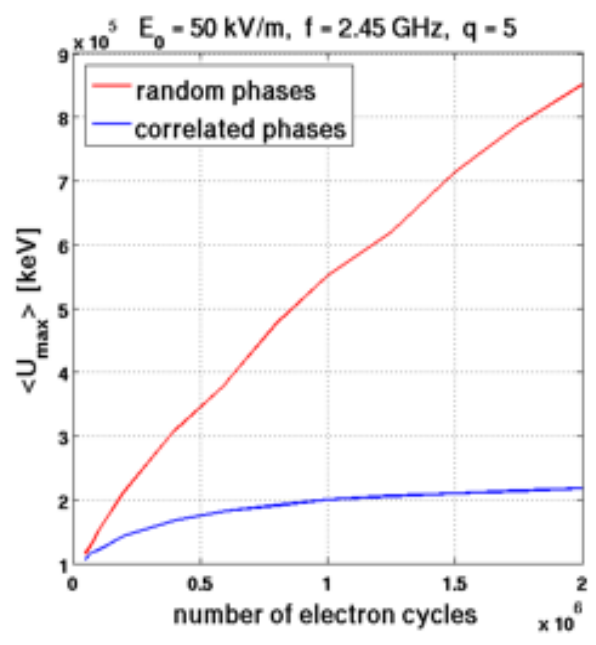

FIG. 8. Average maximum energy $\left\langle\mathrm{U}_{\max }\right\rangle$ in an ensemble of 1000 electrons distinguished initially by random phases but all having an identical initial energy $50 \mathrm{keV}$.

\section{SYMPLECTIC INTEGRATIONS OF EQUATIONS OF MOTION}

In order to ascertain that the simple F-U model captures the essence of the WEGA lower hybrid interaction, we now corroborate its results by numerical integration of equation of motion (2), which describes electron motion through the non-zero width antenna field region with electric field $E_{z}(z, t)=\operatorname{Re}\left[E_{z}(z)\right] \sin (\omega t)$ of FIG. 2a, determined by the full wave coupling code of Preinhaelter ${ }^{30}$. Amplitude of the wave was taken to represent input $\mathrm{LH}$ power of $10 \mathrm{~kW}$. We take a non-zero electric field only in the interaction region of width $d=0.31 \mathrm{~m}$ and treat electrons as free particles on the rest of their drift orbits of length $\mathrm{L}_{\text {free }}=\mathrm{L}-\mathrm{d}=22.31 \mathrm{~m}$. The integration was carried out with a $4^{\text {th }}$ order symplectic method ${ }^{32}$, verified with the Runge-Kutta-Fehlberg scheme $^{33}$.

First, we analyze the change of particle momentum $\mathrm{p}_{1}-\mathrm{p}_{0}$ after one particle orbit. Integration results of FIG. 9a 

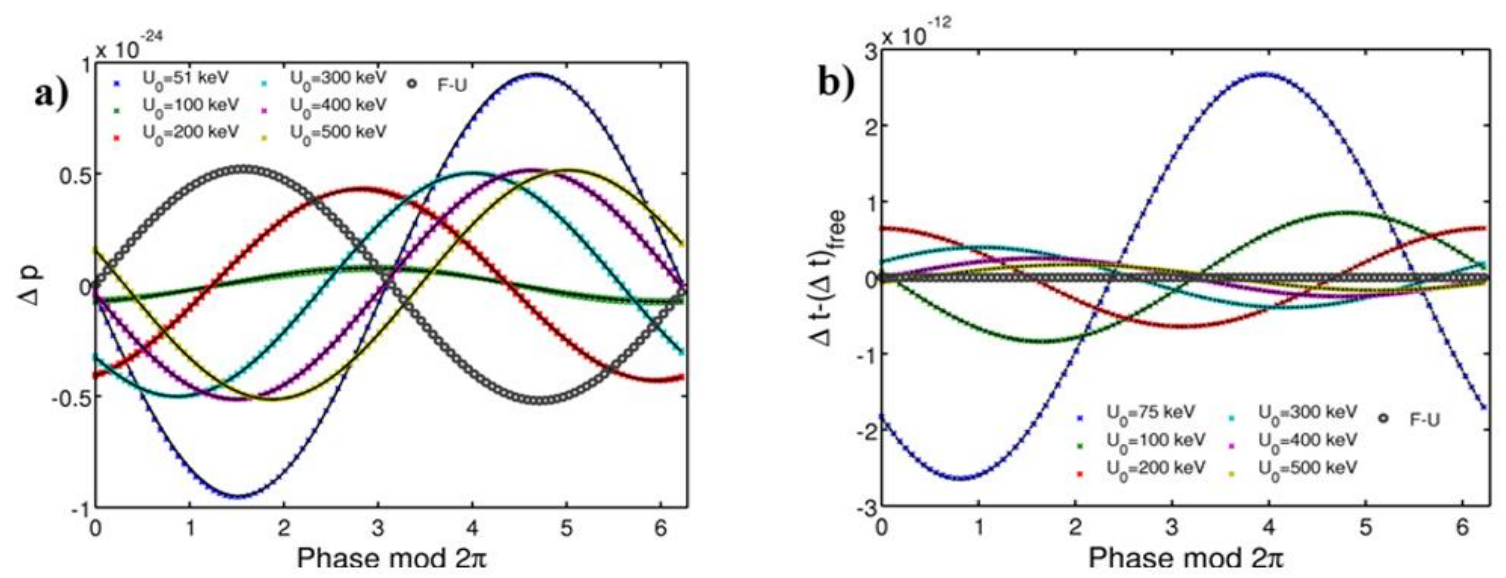

FIG. 9. a) Change of momentum $\Delta \mathrm{p}=\mathrm{p}_{1}-\mathrm{p}_{0}$ and b) modulation of orbit time with respect to orbit time of free particle $(\Delta \mathrm{t})_{\text {free }}$, for the case of particles interacting with the standing wave of FIG. 2a. The fit of relations (7) and (9) for each initial energy is indicated by black lines.

show that momentum exchange between particle and the wave, for electron energies above 50 $\mathrm{keV}$, can be described by

$$
\Delta \mathrm{p} \equiv \mathrm{p}_{1}-\mathrm{p}_{0}=\mathrm{A}_{\mathrm{p}}\left(\mathrm{p}_{0}\right) \sin \left(\omega \mathrm{t}_{0}+\phi_{\mathrm{p}}\left(\mathrm{p}_{0}\right)\right)
$$

This relation resembles the first equation in the F-U mapping (3), but with momentum amplitude dependent on the momentum exchange $A_{p}$ and its phase $\phi_{p}$. FIG. 9a shows how $\Delta p$ depends on the initial phase of the particle in the wave, computed for several selected values of $p_{0}$. An excellent agreement between the relation (7) and numerical integration verifies that the momentum exchange described by a sine dependence on the phase, as assumed in the F-U model, can be used to describe local (in the sense of constant $\mathrm{p}_{0}$ ) particle-wave interaction also for the case of full $\mathrm{E}_{\mathrm{z}}(\mathrm{z}, \mathrm{t})$ profile. The scan shown in FIG. 10 over a range of $\mathrm{p}_{0}$ values shows dependence of $A_{p}$ and $\phi_{p}$ on the initial momentum. 

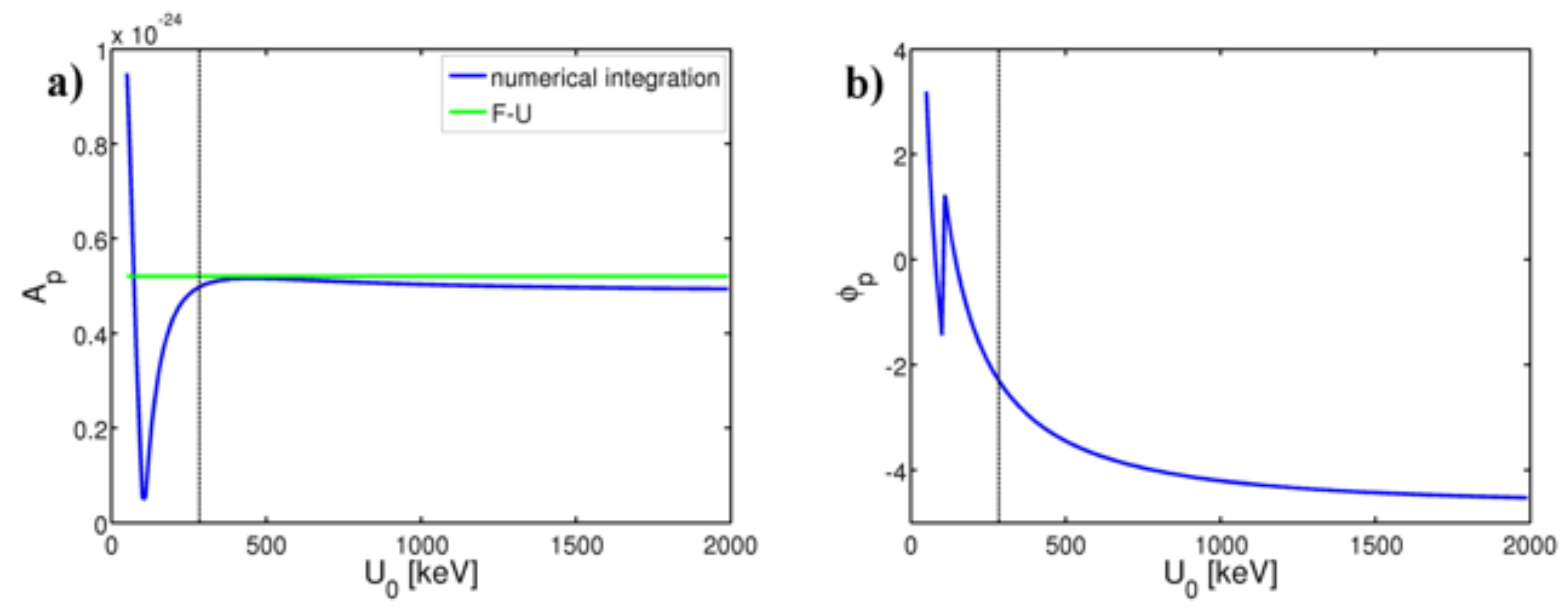

FIG. 10. a) Amplitude of momentum exchange $A_{p}$ (blue line) and b) its phase $\phi_{p}$ for the case of particle interacting with the full $\mathrm{E}_{\mathrm{z}}$ profile of the standing wave (FIG. 2a). Green line in the left plot shows momentum exchange in the F-U model. Vertical dotted line marks position of F-U stochasticity threshold.

For energies approximately below $200 \mathrm{keV}$ both $\mathrm{A}_{\mathrm{p}}$ and $\phi_{\mathrm{p}}$ are strongly dependent on the particle energy and the global F-U map is not expected to provide correct results in this region. For higher energies, however, $A_{p}$ and $\phi_{p}$ converge to constant values and the particle dynamics is well represented by the F-U map. We note that the value of $\mathrm{E}_{0}$ used in the previous section in the F-U map (3) shows good agreement as regards the value of momentum exchange in FIG. 10a in the region around $300 \mathrm{keV}$ where the stochastic threshold is expected. The most pronounced difference between the interaction with a realistic spatial profile of $E_{z}$ and the $\delta$-function approximation in the F-U model is a strong reduction of energy exchange observed around 106 $\mathrm{keV}$ (as seen in FIG. 10a). This region corresponds to the particle energy for which its transit time $\Delta t$ through the non-zero part of $E_{z}$ is comparable to the field oscillation period $T=1 / f_{L H}$. When this condition is satisfied, then as a consequence of field shape and symmetry the particle gains and loses similar amounts of energy in the first and second half of the interaction and its momentum exchange with the wave is strongly reduced, as can be seen in FIG. 11. 

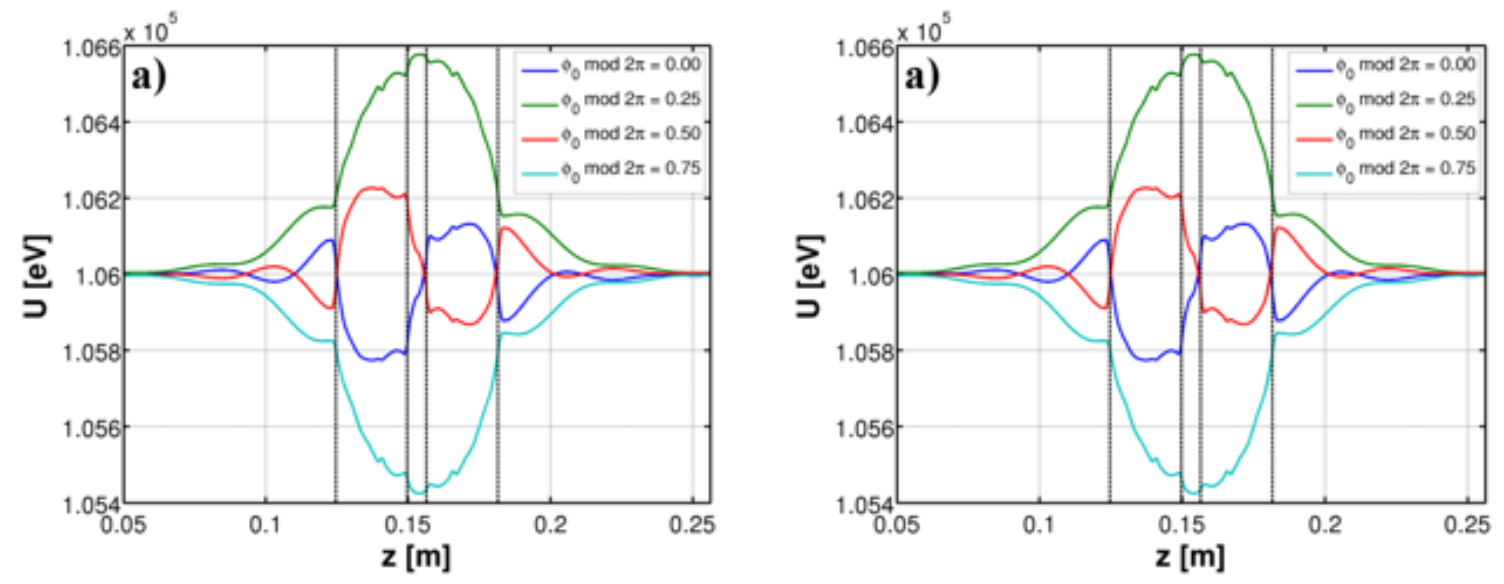

FIG. 11. a) Evolution of particle kinetic energy and b) electric field acting on particle during its transit through the wave for the case of resonant particles with $\Delta t \approx 1 / f$. Four different initial phases $\phi_{0}$ of the particle are shown. Position of the four main peaks of $E_{z}$ is marked by black dashed vertical lines.

As the shape of the field indicates, the effect of the $E_{z}$ field on a particle can be approximated by several separated kicks corresponding to the main peaks of the field

$\Delta \mathrm{p} \approx \delta \mathrm{p}_{1} \sin \left(\omega\left(\mathrm{t}-\mathrm{d}_{1} / 2 / \mathrm{v}\right)\right)+\delta \mathrm{p}_{2} \sin (\omega \mathrm{t})+\delta \mathrm{p}_{1} \sin \left(\omega\left(\mathrm{t}+\mathrm{d}_{1} / 2 / \mathrm{v}\right)=\sin (\omega \mathrm{t})\left(\delta \mathrm{p}_{2}+2 \delta \mathrm{p}_{1} \cos \left(\omega \mathrm{d}_{1} /(2 \mathrm{v})\right)\right)\right.$

where $\delta \mathrm{p}_{1}$ and $\mathrm{d}_{1}$ represent respectively the amplitude of the kick given by the two major peaks on the wings of $E_{z}$ and their distance from the center of the wave, and $\delta p_{2}$ is the amplitude of the joint kick given by the two minor peaks in the middle of the field. Here we assume that the change of particle velocity in the field is negligible compared to particle's initial velocity $\mathrm{v} \approx \mathrm{d} / \Delta \mathrm{t}$. Then the condition $\Delta \mathrm{p}=0$ translates as

$$
2 \delta \mathrm{p}_{1} \cos (\omega \delta /(2 \mathrm{v}))+\delta \mathrm{p}_{2}=0 .
$$

This shows that a similar reduction of the energy exchange should be observed also for higher resonances (located below the initial $50 \mathrm{keV}$ ) as is verified for the case of $\Delta \mathrm{t} \approx 2 / \mathrm{f}_{\mathrm{LH}}$ in FIG. 15 . 
In the range of energies $93-128 \mathrm{keV}$, i.e. around the first-order resonance, the stochastic sea is temporarily replaced by regular motion, as seen in FIG. 12
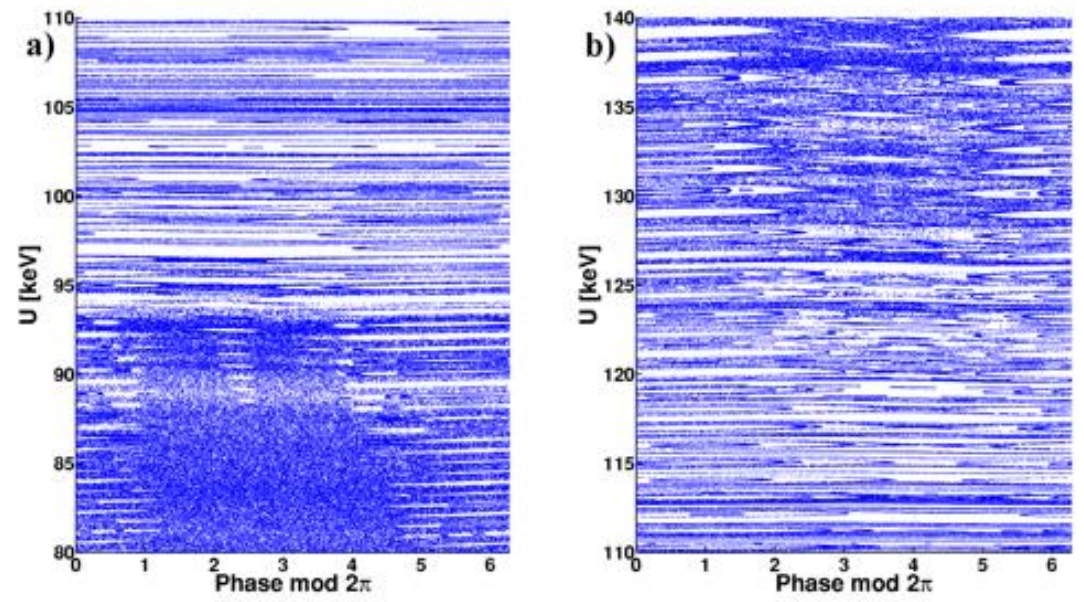

FIG. 12. Poincaré sections of 1500 particles at the entrance to the interaction region. Particles were initially distributed randomly in the range $80-200 \mathrm{keV}$ and followed for 1000 orbits. a) Stochastic sea reaches up to $93 \mathrm{keV}$, and b) a new stochastic region is formed above $128 \mathrm{keV}$.

Above $128 \mathrm{keV}$ the dynamics becomes stochastic again and the stochastic sea extends up to 258 $\mathrm{keV}$ where a transition to regular dynamics starts. For such energies the F-U process already well-represents the dynamics and therefore the global stochastic energy threshold predicted by the F-U model, $\mathrm{E}_{\mathrm{thr}, \mathrm{F}-\mathrm{U}}=284 \mathrm{keV}$, is close to the energy $\mathrm{E}_{\mathrm{thr}, \text { full }}=258 \mathrm{keV}$ of transition to regular motion found numerically for the full profile of $E_{z}$, estimated from FIG. 13.

We note that both values scale similarly with increasing amplitude of the electric field $\left(\mathrm{E}_{\mathrm{thr}, \mathrm{F}-\mathrm{U}}=\right.$ $489 \mathrm{keV}$ and $\mathrm{E}_{\text {thr,full }}=474 \mathrm{keV}$ for an amplitude 2.5 times higher, and $\mathrm{E}_{\mathrm{thr}, \mathrm{F}-\mathrm{U}}=705 \mathrm{keV}$ and $\mathrm{E}_{\text {thr,full }}=685 \mathrm{keV}$ for an amplitude 5 times higher) and the F-U prediction remains within bounds of a $10 \%$ relative error. 

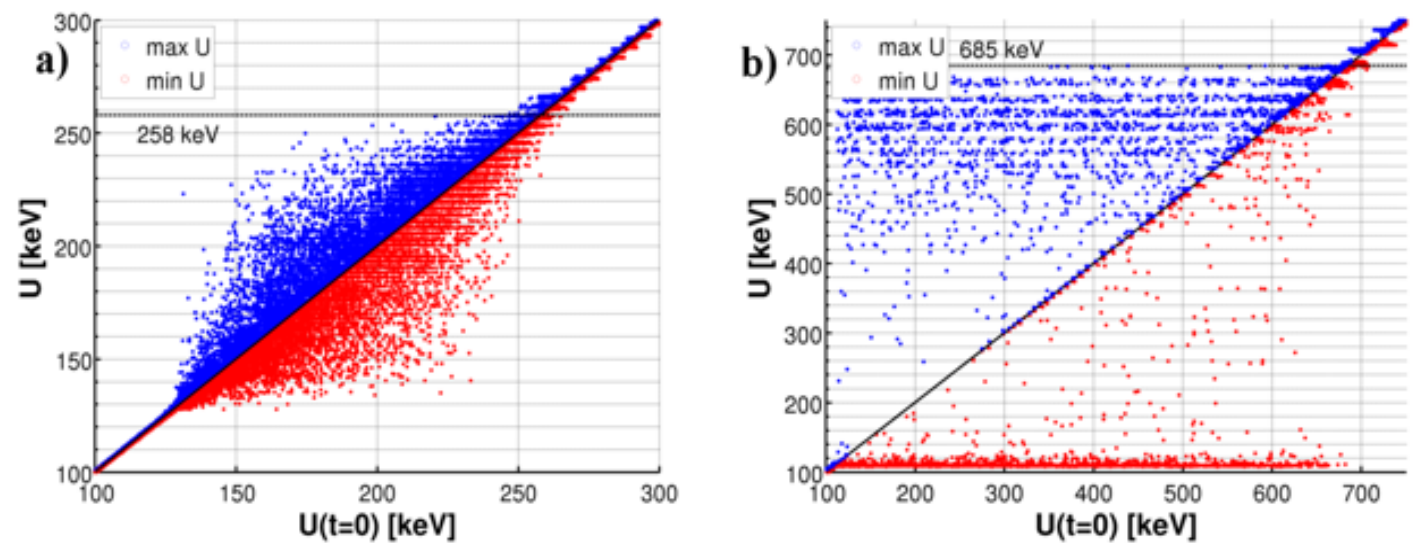

FIG. 13. a) Minimum and maximum energy of particles for the case of standing wave from FIG. 2a and b) using the same field with 5x higher amplitude after 500000 orbits as a function of their initial energy. Initial phase of the particles was chosen randomly. Black horizontal line shows estimated position of the stochasticity threshold.

As shown in FIG. 9b the period of a complete orbit $\Delta \mathrm{t}$ is given as the orbit period of a free particle $\Delta \mathrm{t}_{\text {free }}=\mathrm{Lm}_{\mathrm{e}} \gamma_{1} / \mathrm{p}_{1}$, modulated by an additional oscillating term,

$$
\Delta \mathrm{t}=\mathrm{t}_{1}-\mathrm{t}_{0}=\mathrm{Lm}_{\mathrm{e}} \gamma_{1} / \mathrm{p}_{1}+\mathrm{A}_{\mathrm{t}}\left(\mathrm{p}_{0}\right) \sin \left(\omega \mathrm{t}_{0}+\mathrm{f}_{\mathrm{t}}\left(\mathrm{p}_{0}\right)\right)
$$

A fit of the values $A_{t}$ and $\phi_{t}$ as a function of particle energy is plotted in FIG. 14.
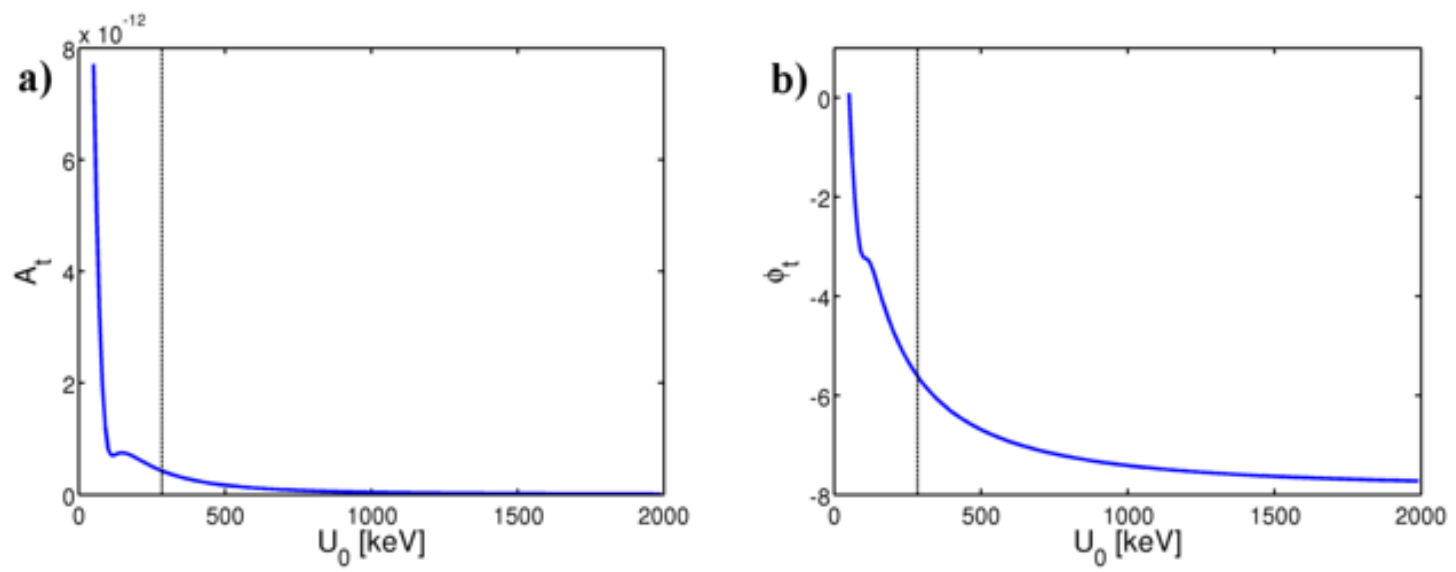
FIG. 14. a) Amplitude of orbit time modulation $A_{t}$. b) Phase $\phi_{t}$ for the case of particles interacting with the full $\mathrm{E}_{\mathrm{z}}$ profile of the standing wave (FIG. 2a). Vertical dotted line shows position of F-U stochasticity threshold.

Since the characteristic relative change of particle velocity in the wave is small, the second term in (8) is negligible compared to the first one. Moreover, since $A_{t}\left(p_{0}\right)<<1 / f_{L H}$, the second term in (8) does not significantly alter the particle phase and can be neglected. The remaining term is identical with the second equation of the F-U model (3) which thus represents a first-order approximation of the particle orbit time.

As was already pointed out, the particles can cross the non-stochastic regions of phase space and be accelerated above the stochastic threshold only if the electron phase between consecutive interactions is uncorrelated. In such a case the particle undergoes diffusion in velocity space with the diffusion coefficient

$$
\left.\mathrm{D}\left(\mathrm{U}_{0}\right)=\left\langle\left(\mathrm{U}_{1}-\mathrm{U}_{0}\right)^{2}\right\rangle /\left(2<\mathrm{t}_{\text {bounce }}\right\rangle\right)
$$

shown in FIG. 15:

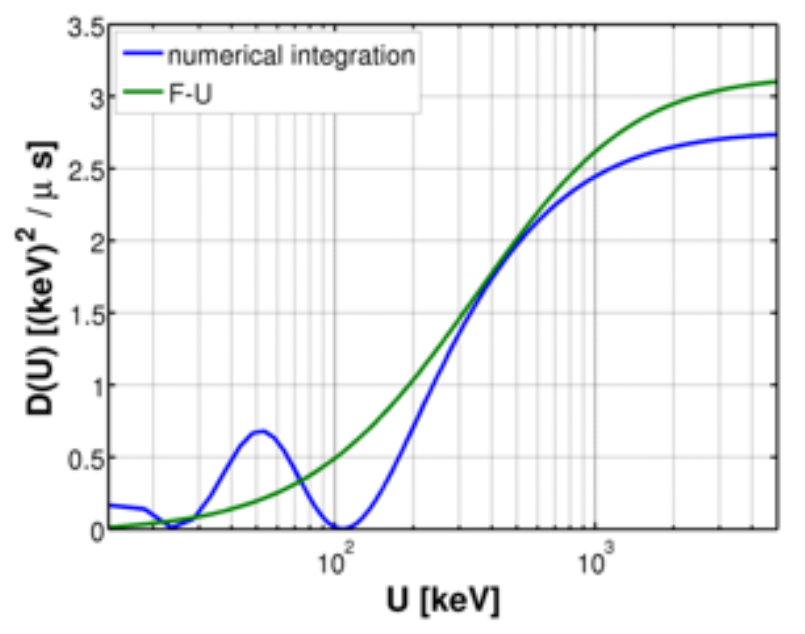

FIG. 15. Diffusion coefficient from Eq.(9), for the case of the full $\mathrm{E}_{\mathrm{z}}$ profile, in blue, and from the F-U map, in green.

Here $\mathrm{U}_{0}$ and $\mathrm{U}_{1}$ are kinetic energy before and after interaction, respectively, and tbounce is period of particle's orbit. The diffusion coefficient saturates for energies in the $\mathrm{MeV}$ range and in 
agreement with the F-U model its value is sufficient to accelerate electrons up to $2.5 \mathrm{MeV}$ in the time frame of the WEGA experiment ${ }^{1}, t_{\text {LHW }} \cong 6.5 \mathrm{~s}$.

\section{SUMMARY AND CONCLUSION}

To summarize, this paper is made up of two main themes. The first is a high-energy relativistic generalization of the so-called simplified Fermi-Ulam (F-U) map, the second is its application to WEGA stellarator lower hybrid (LH) power operation ${ }^{1}$. The simplified F-U map describes a particle bouncing between two stationary walls, one active and one passive. The active wall imparts momentum to the particle, which then returns to this wall after bouncing off the passive one. The map does not formally change if one removes the passive wall and lets the particle circulate, returning to the active wall. This is the situation experienced by electrons on drift surfaces near the antenna during WEGA LH operation. The role of the active wall is here assumed by the standing wave pattern of the WEGA antenna $E_{z}$ field. We essentially demonstrate that an interfering random phase between interaction events is necessary for electron acceleration beyond a global stochastic limit which holds for correlated phases. It is a curious fact that even for very infrequent randomizing events (we tried, for example, randomizing the phase on every $10000^{\text {th }}$ orbit out of $10^{6}$ orbits) the particle energy keeps increasing. In the fluctuating edge plasma such occasional randomizing events can be expected to occur. Moreover, since our model is constructed as 1D, effects of perpendicular particle energy should in the lowest approximation contribute to render the particle phase stochastic.

In order to represent the electron dynamics [equations of motion (1) and (2) and FIG. 2a] by the F-U map, the "real" field region of non-zero extent must be contracted to a $\delta$-function. This can be done if the particle transit time through the field region is shorter than the field oscillation period. Numerical symplectic integrations of the equations of motion in section IV with the "real" antenna field then show for higher electron energies excellent agreement with results from the F-U model. This indicates that the F-U model is a good generic representation of the dynamics of sufficiently energetic particles in a spatially complicated oscillating field. Indeed, for particle energies below $200 \mathrm{keV}$ the F-U process does not correctly represent the interaction and a new type of resonance between the particle transit time through the wave and the field 
oscillation period appears, decreasing the diffusion coefficient in a narrow region around the resonant energy.

There appear to be two main factors allowing the generation of $\mathrm{MeV}$ electrons and noninductive current during LH operation with an un-phased antenna on WEGA. First, the $50 \mathrm{keV}$ electrons generated during EC pre-heating suffer sufficiently few collisions in order to allow them to circulate around drift surfaces without slowing down, thereby gaining energy by repeated interaction with the field at the antenna mouth. In doing so, the electrons become even less collisional. This is a typical runaway situation, taking place when the fast electron meanfree-path is not smaller than the total orbiting path. Second, since motion on the drift surfaces is asymmetric, such that electrons in one direction are not confined ${ }^{1-3}$, a current is generated.

In the case of correlated phases between interaction events, we find that the electron energy saturates with the number of passes through the interaction region. The energy saturation values from iterations of the F-U map is found to agree with values from period-1 fixed point stability analysis. These results are confirmed by direct numerical integration with a finite width interaction region. In contrast, when the phases between interaction events are assumed random, the electron energy obtained from F-U simulations does not saturate with the number of passes. This result is again corroborated by numerical integration with a finite-sized antenna field region. The numerical results for random phases, for example the diffusion coefficient of FIG 15, essentially corroborate the result of Laqua et al. ${ }^{1}$. The iterated F-U map can also yield global quantities of interest such as maximum energy ensemble averages shown in FIG. 8, revealing that with random phases intervening between interaction events, fast electrons in the MeV range can be expected to be generated within the time frame of the WEGA experiment ${ }^{1}, t_{L H W} \cong 6.5 \mathrm{~s}$.

The present relativistic form of the F-U map could naturally have useful astrophysical applications, cosmic radiation being the area for which the $\mathrm{Fermi}^{7}$ map was originally formulated.

\section{ACKNOWLEDGMENTS}

We thank Prof. R. A. Cairns for valuable comments. This work was partly supported by MSMT Project LM2011021 and by GACR grants 13-38121P and GAP205/10/2055. We greatly appreciate the access to the CERIT-SC computing and storage facilities provided under the 
program Center CERIT Scientific Cloud, part of the Operational Program Research and Development for Innovations, reg. no. CZ. 1.05/3.2.00/08.0144, as well as access to computing and storage facilities owned by parties and projects contributing to the National Grid Infrastructure MetaCentrum, provided under the program "Projects of Large Infrastructure for Research, Development, and Innovations" (LM2010005).

*This is an extended version of the contribution "Lower Hybrid Wavepacket Stochasticity Revisited", presented at the $20^{\text {th }}$ Topical Conference on RF Power in Plasmas, 25-29 June, Sorrento, Italy.

${ }^{1}$ H. P. Laqua, E. Chlechowitz, M. Otte and T. Stange, submitted to Plasma Physics and Controlled Fusion. ${ }^{2}$ M. Otte, H. P. Laqua, E. Chlechowitz, S. Marsen, J. Preinhaelter, T. Stange, A. Rodatos, J. Urban and D. Zhang, Nukleonika, 57 (2012) 171-175.

${ }^{3}$ M. Otte, H. P. Laqua, E. Chlechowitz, P. Drewelow, M Glaubitz, S. Marsen, E. Müller, Y. Podoba, J. Schacht, T. Stange, F. Steffen, F. R. Wagner and D. Zhang, Contributions to Plasma Physics, 50 (2010) 780.

${ }^{4}$ M. A. Lieberman and A. J. Lichtenberg, Phys. Rev. A5 (1972) 1852.

${ }^{5}$ A. J. Lichtenberg, M. A. Lieberman and R. Cohen, Physica 1D (1980) 291-305.

${ }^{6}$ E. Fermi, Phys. Rev., 75 (1949) 1169.

${ }^{7}$ E. Fermi, ApJ, 119 (1954) 1.

${ }^{8}$ L. Davis, Phys. Rev., 75 (1956) 351.

${ }^{9}$ R. M. Kulsrud and A Ferrari, Ap. Space Sci, 12 (1971)302.

${ }^{10} \mathrm{~V}$. Petrosian, "Stochastic acceleration by turbulence", Proceedings of ISSI-Bern workshop on Particle Acceleration", 2011; available online as 1205.2136v1 [astro.ph.HE].

${ }^{11}$ A, Chao, D. Johnson, S. Peggs, J. Peterson, C. Saltmarsh, L. Schichinger, R. Meller, R. Siemann, R. Talman and P Morton, Phys. Rev. Lett. 62 (1988) 2752.

${ }^{12}$ C. Chen and R. C. Davidson, Phys. Rev. A42 (1990) 5041.

${ }^{13}$ T. E. Evans, K. H. Burrell, M. E. Fenstermacher, R. A. Moyer, T. H. Osborne, M. J. Schaffer, W.P West, L. W. Yan, J. A. Boedo, E. J. Doyle, G. L. Jackson, I. Joseph, C. J. Lasnier, A. W. Leonard, T. L. Rhodes, P. R. Thomas, J. G Watkins and L. Zeng, Phys. Plasmas 13 (2006) 056121.

${ }^{14}$ Y. Kominis, A. K. Ram and K. Hizanidis, Phys. Rev. Lett. 104 (2010) 23500.

${ }^{15}$ V. Fuchs, V. Krapchev, A. Ram and A. Bers, Physica, 14D (1985) 141.

${ }^{16}$ P. Pavlo and L. Krlin, Plasma Phys. Control. Fusion, 41 (1999) 541.

${ }^{17} \mathrm{R}$. Pánek, $\mathrm{PhD}$ Thesis, "Nonlinear and Stochastic Interaction of Waves with Plasma", Charles University, Prague, (2002).

${ }^{18}$ L. Krlín, P. Pavlo, R. Pánek, R. Klíma and V. Petržílka, Plasma Phys. Control. Fusion, 44 (2002) 159. 
${ }^{19}$ J. Seidl, Master Thesis: "Anomalous diffusion of electrons interacting with a lower hybrid wave in a tokamak“, Charles University, Prague (2006).

${ }^{20}$ J. Mailloux, Y. Demers, V. Fuchs, M. Goniche, P. Jacquet, C. Boucher, A. Cote, C. Cote, J. Gunn, B. Terreault, P. Bibet, P. Froissard, D. Guilhem, J. H. Harris, G. Rey and M. Tareb, J. Nucl. Mater., 241243 (1997) 745.

${ }^{21}$ V. Fuchs, M. Goniche, Y. Demers and J. Mailloux, Phys. Plasmas 3 (1996) 4023.

${ }^{22}$ M. Goniche, D. Guilhem, P. Bibet, P. Froissard, X. Litaudon, G. Rey, Y. Demers, V. Fuchs, P. Jacquet, J. H. Harris and J T Hogan, Nucl. Fusion 38 (1998) 919.

${ }^{23}$ K. M. Rantämaki, T. G. H. Pättikangas, S. J. Karttunen, P. Bibet, X. Litaudon and D. Moreau, Nucl. Fusion 40 (2000) 1477.

${ }^{24}$ J. P. Gunn, V. Petržílka, A. Ekedahl, V. Fuchs, E. Gauthier, M. Goniche, M. Kočan, J.-Y. Pascal and F. Saint-Laurent, J. Nucl. Mater., 390-391 (2009) 904.

${ }^{25}$ V. Petržílka, V. Fuchs, J. P. Gunn, N. Fedorczak, A. Ekedahl, M. Goniche, J. Hillairet and P. Pavlo, Plasma Phys. Control. Fusion 53 (2011) 054016.

${ }^{26}$ B. V. Chirikov, Phys. Reports. 52, (1979) 263.

${ }^{27}$ A. J. Lichtenberg and M. A. Lieberman, "Regular and Stochastic Motion”, Springer-Verlag, New York, 4A, (1983).

${ }^{28}$ L. D. Pustylnikov, Teoretical and Mathematical Physics, 77 (1988) 1110-1115.

${ }^{29}$ Y.Y. Podoba, H. P. Laqua, G. B. Warr, M. Schubert, M. Otte, S. Marsen, F. Wagner and E. Holzhauer, Phys. Rev. Lett. 98 (2007) 255003.

${ }^{30}$ J. Preinhaelter, J. Urban, L. Vahala and G. Vahala, Nucl. Fusion 52 (2012) 083005.

${ }^{31}$ M. Otte, D. Andruczyk, E. Holzhauer, J. Howard, R. Konig, L. Krupnik, H. P. Laqua, O. Lischtschenko, S. Marsen, J. Schacht, J. Urban, Y. Y. Podoba, J. Preinhaelter, F. Wagner, G. B. Warr and A. Zhezhera, AIP Conference Proceedings 993 (2008) 3.

${ }^{32}$ Ernst Hairer, Syvert Nørsett, and Gerhard Wanner, "Solving Ordinary Differential Equations I: Nonstiff Problems", second edition, Springer-Verlag, Berlin (1993).

${ }^{33}$ Erwin Fehlberg, "Klassische Runge-Kutta-Formeln vierter und niedrigerer Ordnung mit SchrittweitenKontrolle und ihre Anwendung auf Wärmeleitungsprobleme," Computing (Arch. Elektron. Rechnen), vol. 6, 1970, pp. 61-71. 